This is an electronic reprint of the original article. This reprint may differ from the original in pagination and typographic detail.

Author(s): Merikoski, Juha

Title: $\quad$ Totally asymmetric exclusion process fed by using a non-Poissonian clock

Year: $\quad 2015$

Version:

Please cite the original version:

Merikoski, J. (2015). Totally asymmetric exclusion process fed by using a non-

Poissonian clock. Physical review E, 91(6), Article 062101.

https://doi.org/10.1103/PhysRevE.91.062101

All material supplied via JYX is protected by copyright and other intellectual property rights, and duplication or sale of all or part of any of the repository collections is not permitted, except that material may be duplicated by you for your research use or educational purposes in electronic or print form. You must obtain permission for any other use. Electronic or print copies may not be offered, whether for sale or otherwise to anyone who is not an authorised user. 


\title{
Totally asymmetric exclusion process fed by using a non-Poissonian clock
}

\author{
Juha Merikoski* \\ Department of Physics, University of Jyvaskyla, P.O. Box 35, FI-40014 University of Jyvaskyla, Finland
}

(Received 22 October 2014; revised manuscript received 15 January 2015; published 3 June 2015)

\begin{abstract}
In this article we consider the one-dimensional totally asymmetric open-boundary exclusion process fed by a process with power-law-distributed waiting times. More specifically, we use a modified Pareto distribution to define the jump rate for jumps into the system. We then characterize the propagation of fluctuations through the system by kinetic Monte Carlo simulations and by numerical evaluation of the steady-state partition function.
\end{abstract}

DOI: 10.1103/PhysRevE.91.062101

PACS number(s): 05.40.-a, 02.50.Ey, 64.60.an

\section{INTRODUCTION}

The totally asymmetric exclusion process (TASEP) and the related zero-range process have become paradigmatic models of low-dimensional nonequilibrium dynamics [1-6]. Macroscopic quantities of interest, in particular the particle density and current and their fluctuations as well as configurational properties, such as the dynamics of the largest cluster and the condensation transition, have been of renewed interest for various generalizations [7-21] of the basic TASEP model, many of them interesting, e.g., from the point of view of RNA transcription, jamming of traffic flow, phase separation, and growth phenomena.

One generalization of the TASEP studied recently is the model "with finite resources" where several TASEP chains compete for a fluctuating number of incoming particles available because of restricting the total number of particles in the system [22]. This is motivated by a biological application where the number of ribosomes available fluctuates, see also Ref. [14]. Another modification of input rates is periodic driving [23]. Also the fully non-Poissonian exclusion process has been investigated [24]. In this article we consider a single TASEP where the chain is fed with the jump rate of incoming particles being non-Poissonian with a wide distribution of waiting times for a new particle to come in. Below we call the TASEP with this modification the fed TASEP (FTASEP). In practical applications, the reason for such a waiting time distribution might be fluctuations of the number and density of particles available or the chemical potential due to other processes in the environment.

\section{THE SIMULATION MODEL}

In the standard one-dimensional open-boundary TASEP model there are two rate parameters, the input rate $\alpha$ and the output rate $\beta$ (standard notations) with the rate for jumps in the "bulk" taken to be unity. The site occupation numbers are denoted by $n_{i}$, where $i=1, \ldots, L$ can have values $n_{i}=0,1$ for empty and occupied sites, respectively, and $L$ is the size of the system.

In the FTASEP the constant input rate $\alpha$ into site $i=1$ of the standard TASEP is replaced by

$$
\alpha \rightarrow \alpha(t)=\frac{A}{\xi(t)+\delta},
$$

\footnotetext{
*juha.t.merikoski@jyu.fi
}

where the random variable $\xi$ is drawn from a modified Pareto distribution,

$$
f(\xi)=\gamma a(1+\gamma \xi)^{-a-1},
$$

where the decay of the distribution is determined by the parameter $a$. The rate $\alpha(t)$ (and the related expected waiting time) is defined when the lattice site $p=1$ becomes empty and it keeps its value until the site gets occupied. The other parameters $A, \gamma$, and $\delta$ are constants. Note that for the decay parameter we use here notation $a$ instead of notation $\alpha$, which is reserved for standard use in the context of exclusion processes.

In this paper we choose $\gamma=\delta=1$. This leads for $A=1$ to input rate statistics, where

$$
\left\langle\frac{1}{\xi+1}\right\rangle=\frac{a}{1+a}
$$

and

$$
\left\langle\left(\frac{1}{\xi+1}\right)^{2}\right\rangle-\left\langle\frac{1}{\xi+1}\right\rangle^{2}=\frac{a}{(1+a)^{2}(2+a)} .
$$

The corresponding effective waiting times are determined by the inverse quantities,

$$
\langle\xi+1\rangle=\frac{a}{a-1},
$$

valid and finite for $a>1$, and

$$
\left\langle(\xi+1)^{2}\right\rangle-\langle\xi+1\rangle^{2}=\frac{a}{(a-1)^{2}(a-2)},
$$

finite for $a>2$.

It turns out that for small values of $a$ because of the diverging waiting times, there is no reasonable way to choose the constant $A$ so that the input rate would correspond to a given constant rate for all values of $a$. Since arbitrarily long waiting times are possible, there are time epochs when the lattice, no matter how large, is empty and the observed fluctuation statistics will depend on the length of the simulation. However, by choosing $A=(1+a) / a$ the mean input rate is constant and

$$
A=\frac{1+a}{a} \Rightarrow\left\langle\frac{A}{\xi+1}\right\rangle=1 .
$$

For increasing $a$ well above 2, the dynamics of the FTASEP approaches that of a TASEP with a constant input rate. On the other hand, for small $a$ the average waiting time diverges.

Since the average values of the density and current in the standard TASEP are $\rho=1 / 2$ and $J=1 / 4$ in the maximal 
current phase, long waiting times in the FTASEP for small $a$ lead to a situation, where $\rho=1 / 2$ and $J=1 / 4$ are beyond reach regardless of the choice of the parameter $A$ as the TASEP dynamics of the bulk restricts the current for times during time epochs when there are particles in the lattice.

In the simulations of this model the dynamics is generated by using a rejection-free continuous-time kinetic Monte Carlo method [25,26] by which we can handle arbitrarily long (and short) waiting times. For the TASEP simulations we choose input and output rates $\alpha=\beta=1$ for which there exist analytical results in the literature, see, e.g., Refs. [3,9]. For the FTASEP we choose the Pareto parameter values $\gamma=\delta=1$ varying the decay parameter $a$ with $A=(1+a) / a$ and $\beta=1$ to allow comparison with the TASEP.

\section{RESULTS FOR LOCAL DENSITY FLUCTUATIONS BY SIMULATIONS}

First, we compare the density fluctuations for the FTASEP with those reported for the TASEP by Derrida et al. in Ref. [9], in particular the exact analytical results in their seminal article. Following their approach, which facilitates the comparison of analytical and numerical results for the TASEP, we calculate the quantities $s^{2}(x)$ and $s^{4}(x)$, essentially the variance and skewness for local density fluctuations.

For direct comparison with their results, in Fig. 1 we use their division of the lattice into intervals such that the value of parameter $x$ corresponds to an interval $L(1-x) / 2 \leqslant i \leqslant$ $L(1+x) / 2$, where $i$ is the index of the lattice site. With this choice, for increasing $x \equiv 2 k / L$ with $k=1,2, \ldots, L / 2$, the interval expands symmetrically from the middle of the system such that for $x=1$ the interval covers whole system. For a such division of the lattice, Derrida et al. [9] give for $s^{2}(x)$ and $s^{4}(x)$ in the TASEP analytical formulas [see Eq. (3.7-8) in their paper], and they test them by simulations of the TASEP [see Fig. 1 in their work]. In Fig. 1 we see how the behavior of these quantities for the FTASEP for small values of $a$ considerably differ from those for the TASEP. (Note that the vertical scale in our plot is logarithmic.) For increasing $a$, the results for the FTASEP approach those for the TASEP.

Since in the choice above of intervals as in Ref. [9], the interval expands symmetrically from the middle of the system (which for symmetry reasons is optimal for the analytical study of the TASEP), it does not tell about the differences between the two halves of the lattice (relevant for the FTASEP). Therefore, we next calculate in Fig. 2 the quantities $s_{A}^{j}(x)$ and $s_{B}^{j}(x)$ characterizing density fluctuation statistics separately $(A)$ for the input half of the lattice and $(B)$ for the output half of the lattice.

For $s_{A}^{2}(x)$ and $s_{A}^{4}(x)$ the intervals are parametrized as $L(1-x) / 2 \leqslant i \leqslant L / 2$, whereas for $s_{B}^{2}(x)$ and $s_{B}^{4}(x)$ they are $L / 2+1 \leqslant i \leqslant L(1+x) / 2$ where, as before, $0<x<1$ and $k=1,2, \ldots, L / 2$. For increasing $x$, in case $(A)$ the interval thus expands from the middle of the lattice towards the input site $i=1$, and in case $(B)$ the interval expands from the middle towards the output site $i=L$. It turns out that using intervals of increasing length provides a convenient way to compare the fluctuations at the input and output ends of the lattice as follows: In Fig. 2 we show the ratios $s_{A}^{j}(x) / s_{B}^{j}(x)(j=2,4)$.
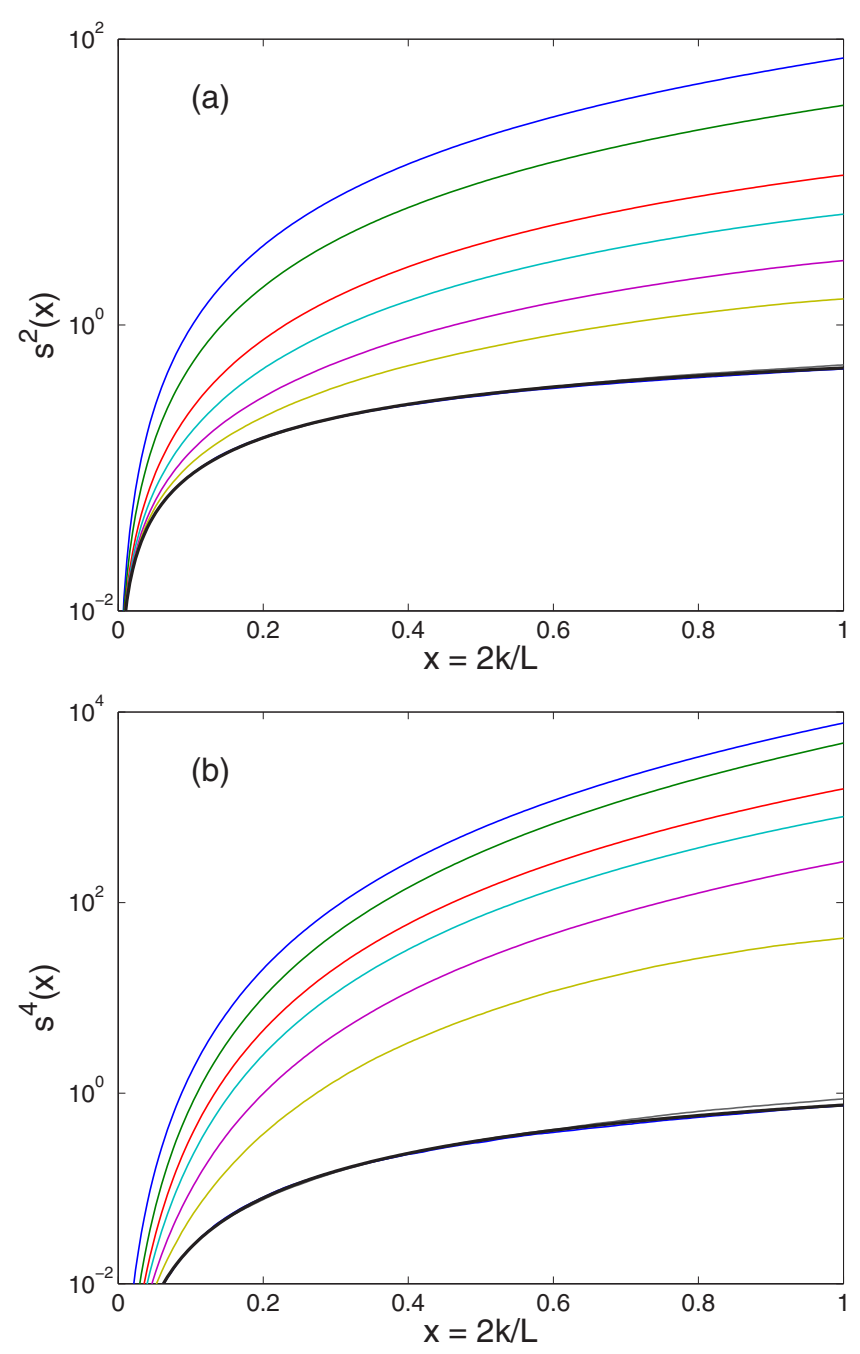

FIG. 1. (Color online) Simulation data for (a) $s^{2}(x)$ and (b) $s^{4}(x)$ for $L=500$. Curves from top to bottom correspond to the FTASEP with $a=1.0,1.2,1.4,1.6,1.8,2.0,3.0,4.0$. The thick black curves are the theoretical results for the TASEP from Ref. [9]. Our data for $a=3.0,4.0$ are hardly discernible from their theoretical curves. In these plots, for increasing $x$ the interval expands symmetrically from the middle of the lattice to allow comparison with the theoretical results of Ref. [9].

Evidently the anomalous fluctuations in the FTASEP are more pronounced in the first half of the lattice, the ratios in general increasing for increasing $a$ and increasing for increasing $x$ (for the TASEP the ratios would be one [9]).

The order of the curves in Fig. 2, i.e., the data for $a=1$ being between data for $a=2$ and $a=3$, reflects the fact that for $a=1$ the waiting time distribution becomes so wide that the averaged particle density is much lower than for $a \geqslant 2$, cf. Eqs. (5) and (6). We will return to this point shortly.

We next turn to the question of what controls local fluctuations in the FTASEP. Obviously the local particle density is an important parameter, but we expect it not to be all there is to say. To study this question, we divide the lattice into ten consecutive intervals of equal length $m=50$ for $L=500$, each with index $p=1, \ldots 10$ corresponding to intervals $(p-1) m+1 \leqslant i \leqslant p m$, and show the scaled 

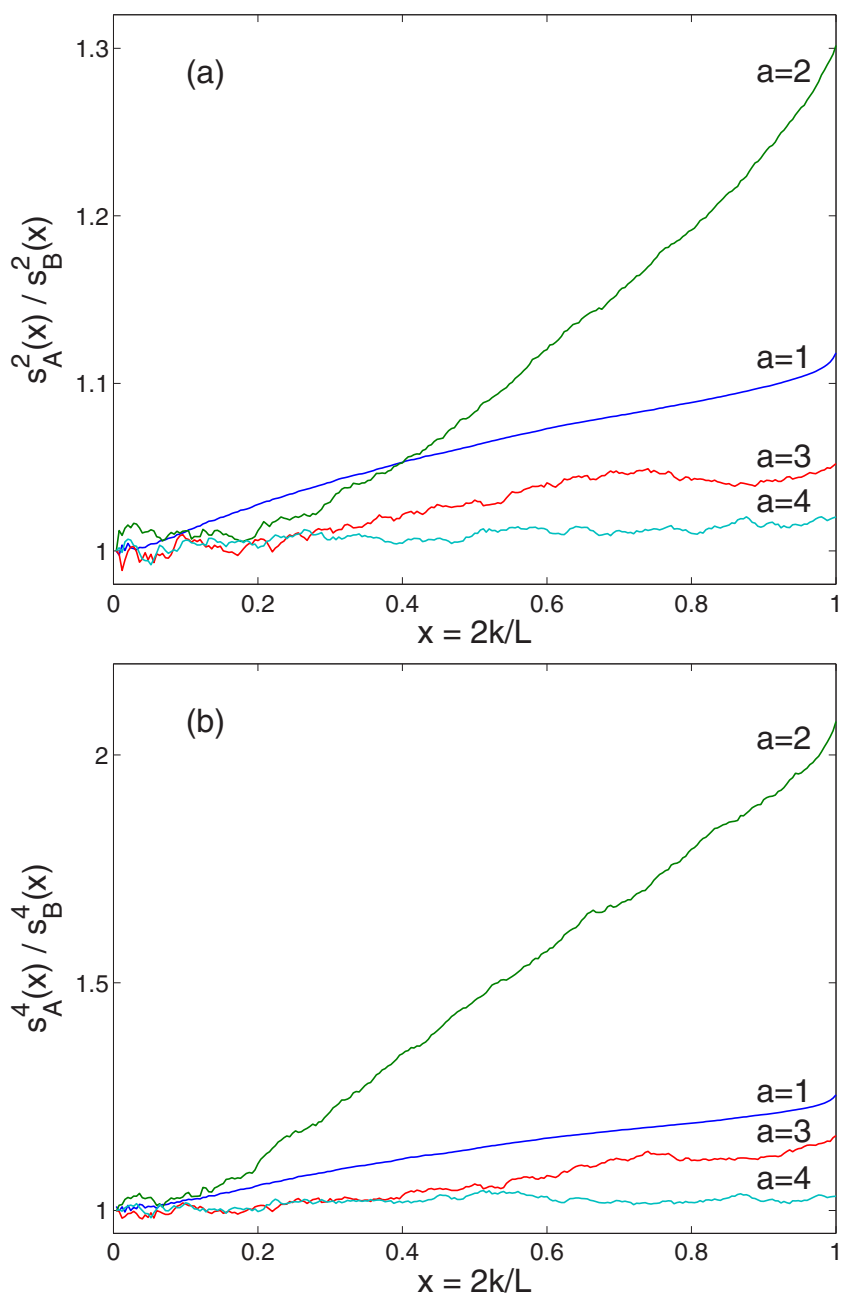

FIG. 2. (Color online) Simulation data for (a) $s_{A}^{2}(x) / s_{B}^{2}(x)$ and (b) $s_{A}^{4}(x) / s_{B}^{4}(x)$ for the FTASEP with $L=500$. For increasing $x$, the intervals expand from the middle of the lattice towards the input end and the output end of the lattice for $A$ and $B$ quantities, respectively.

kurtosis and skewness as a function of the local particle density $\left\langle n_{p}\right\rangle / m$.

First, it is evident that the particle density in the FTASEP tends to increase for increasing $a$, and for large $a$ the density in the middle of the system is close to $1 / 2$. For small $a$, as explained above, there are long epochs of time such that the lattice is empty and the density is very small. This is seen in the behavior of the kurtosis $s^{4} /\left(s^{2}\right)^{2}$ for low densities in Fig. 3(a), which shows that the average fluctuations are completely controlled by the density for $a \leqslant 1$. To make this more evident, we show by the thick black curve the (trivial) single-site occupancy fluctuation statistics corresponding to the given average density.

On the other hand, for $a>1$ the behavior across the system for a given value of $a$ is more complex and, in addition to the local density $\left\langle n_{p}\right\rangle / m$, the value of $a$ becomes relevant as seen for large densities in Fig. 3(b). For very large values of $a$ the value of kurtosis approaches from above the normal distribution limit $s^{4} /\left(s^{2}\right)^{2} \rightarrow 3$. Note that larger values of $\left\langle n_{p}\right\rangle$ correspond to intervals closer to the input site $i=1$ so that each
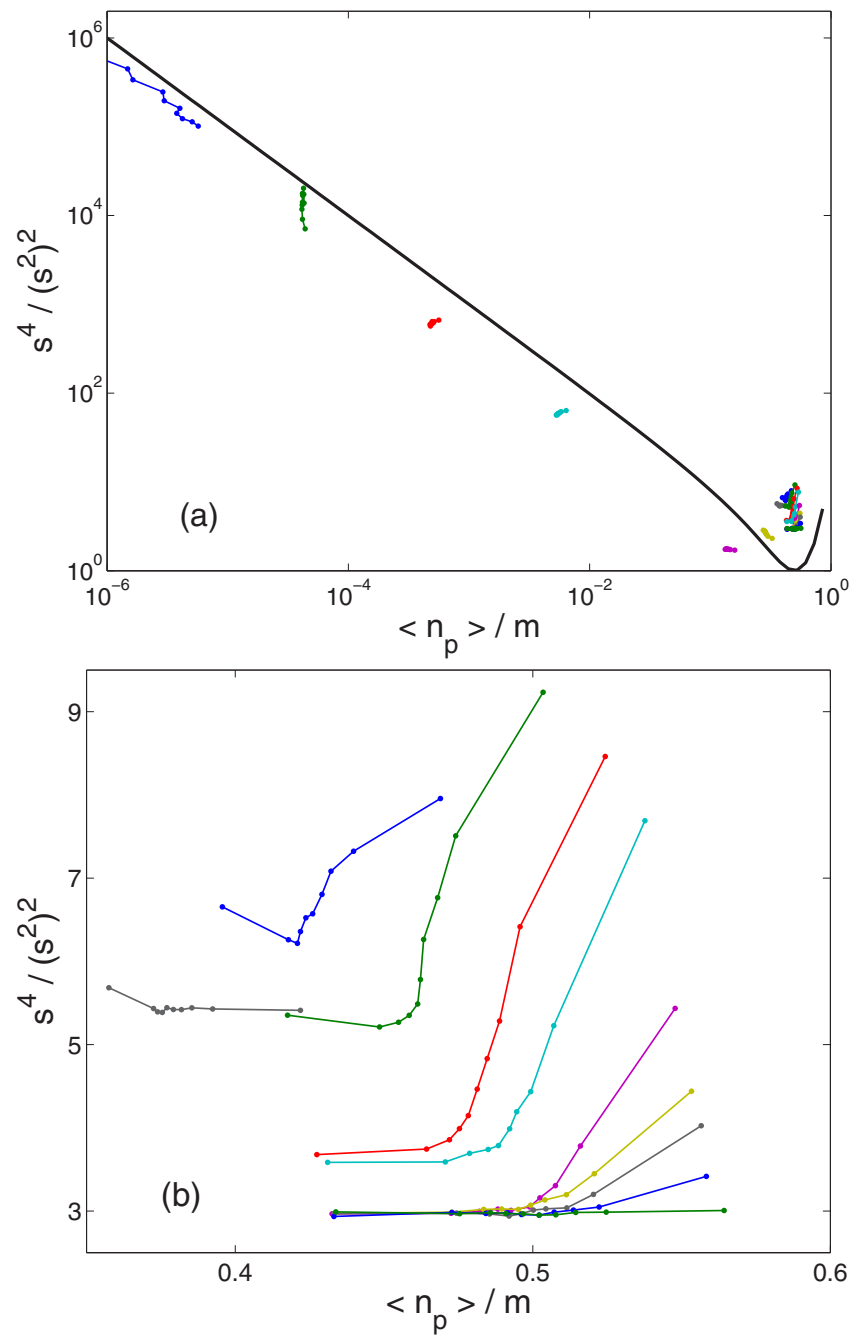

FIG. 3. (Color online) Simulation data for the normalized kurtosis $s^{4} /\left(s^{2}\right)^{2}$ as a function of $\left\langle n_{p}\right\rangle$ for $L=500$ with $m=$ 50. Each curve corresponds to one value of $a$ with $a=$ $0.2,0.4,0.6,0.8,1.0,2.0,2.2,2.4,2.6,2.8,3.0,4.0$ with $a$ increasing from left to right in the plots. Each curve with given $a$ has ten data points with $p=1, \ldots, 10$ increasing from right to left, see the text. Plot (a) displays data for small values of $a$, and plot (b) displays data for large values. However, the data of plot (b) are seen in the lower-right corner of plot (a). The data points show the actual Monte Carlo data; straight lines connecting them are a guide to the eye. The thick black curve shows the single-site approximation described in the text.

curve for a given value of $a$ in Fig. 3 (and in Fig. 4 below) should be read from right to left.

For the data in Fig. 3(b) one can attempt a data collapse. A reasonable one is obtained by scaling the $x$ axis (the horizontal axis) as $x \rightarrow x+(2 / 5)^{a}$ and the $y$ axis (the vertical axis) as $y \rightarrow(y-3)^{(a-1) / 2}$. Because of the unavoidably short span of the data, one might replace $(2 / 5)^{a}$ above by $e^{-a}$. Furthermore, for $a>2$ no shift is needed on the horizontal axis since all the curves in that region start to deviate from the horizontal part $y \approx 3$ around $x=1 / 2$.

For the local skewness $s^{3} /\left(s^{2}\right)^{3 / 2}$, the simulation data across the system shown in Fig. 4 display similar qualitative features. 

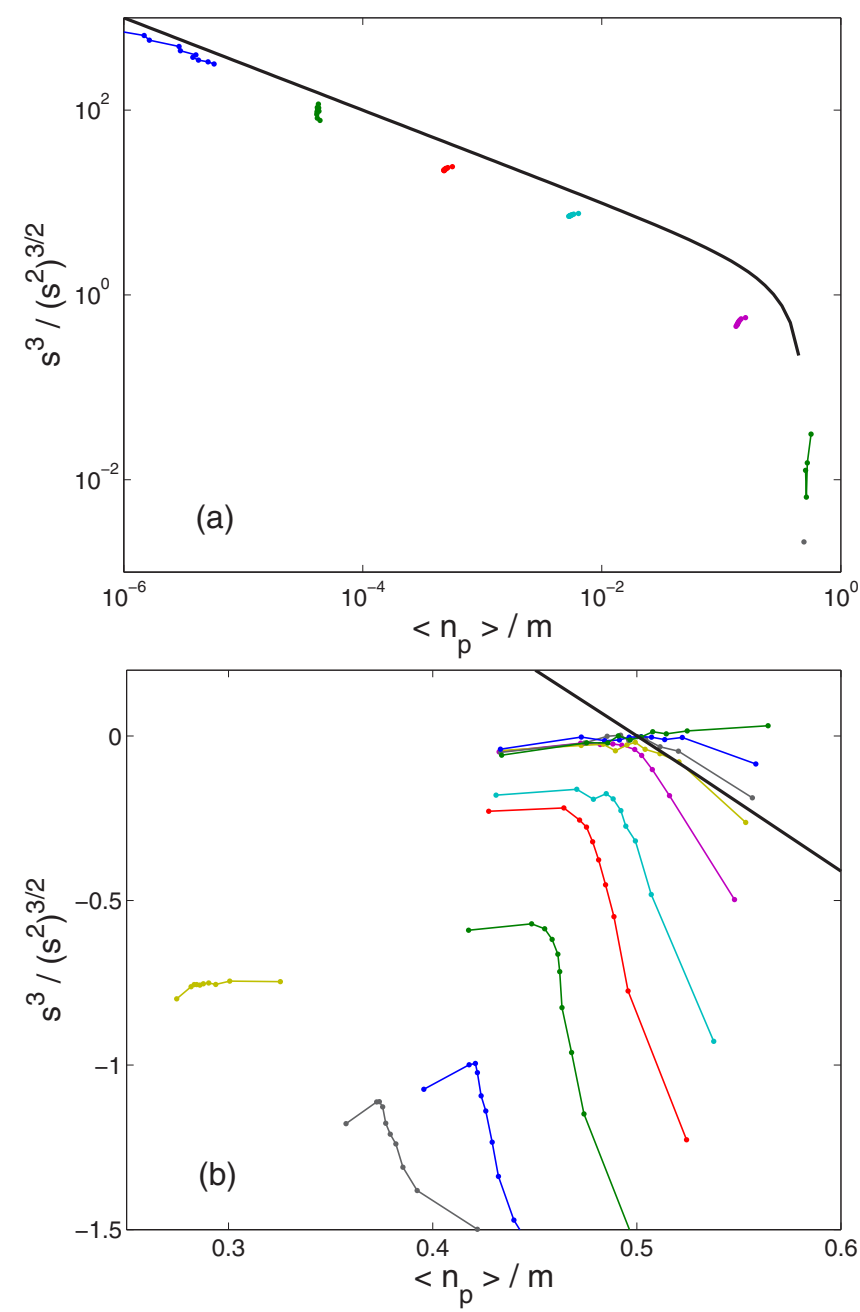

FIG. 4. (Color online) Simulation data for the normalized skewness $s^{3} /\left(s^{2}\right)^{3 / 2}$ as a function of $\left\langle n_{p}\right\rangle$ for $L=500$ with $m=50$. The organization and color coding of data are similar to that in Fig. 3.

For $a \leqslant 1$ [see Fig. 4(a)] they are controlled by the local density and, as expected, for large $a$ [Fig. 4(b)] the skewness approaches the normal distribution value zero from below (weight on the side of large deviations). In both Figs. 3(a) and 4(a), the thick black curves again correspond to the single-site approximation. The simulation data for the FTASEP with small $a$ and, correspondingly, low density $\left\langle n_{p}\right\rangle / m$, follow this prediction closely up to $a=1$. Concerning the details of Figs. 3(b) and 4(b) for intermediate values of $a$, there is again structure in the behavior of the FTASEP such that for a given value of $a$ the skewness is larger in the left end of the lattice (corresponding to large density).

We note that the value $\left\langle n_{p}\right\rangle / m=1 / 2$ deep in the bulk corresponds to the theoretical value for the TASEP with $\alpha=$ $\beta=1[3,9]$. That value of local density is reached in our parametrization of the FTASEP only for $a \geqslant 2$. However, if in the simulations we multiply the input rate coefficient $A=$ $(1+a) / a$ in Eq. (7) by some large factor, then it is possible to reach $\left\langle n_{p}\right\rangle / m \approx 1 / 2$ for smaller values of $\alpha$. Then also, for example, multiplying $A$ by 100 the kurtosis becomes close to three and skewness close to zero for $\alpha \geqslant 1.4$ with $L=500$ (data not shown). The locations of the apparent transitions from a simple behavior for small values of $a$ to a complex behavior for intermediate values of $a$ and then again to a simple behavior for a large value of $a$ also depend on $L$, but the qualitative overall picture remains the same.

\section{THEORETICAL CONSIDERATIONS: THE PARTITION FUNCTION}

Analytical calculations for systems with (part of the) processes obeying the nonexponential clock are challenging. In this section we present one theoretical machinery; an interested reader will find the numerical results obtained by using it for physical quantities in Sec. V.

As a starting point for an effective theory we take the well-known mapping between the TASEP and the Brownian excursions from Ref. [9] by Derrida et al. They show that the TASEP states can be related to random walks with successive steps of length $0, \pm 1$ such that +1 corresponds to an occupied site, -1 corresponds to an empty site, and 0 corresponds randomly to an occupied or an empty site [27].

For this approach, to write the partition function, a few functions need to be defined: First, for the excursion statistics in the TASEP, $y$ and $z$ being two consecutive excursions, we take $[9,27,28]$

$$
\begin{gathered}
F(y, x)=y e^{-y^{2} / x}, \\
G(y, z, x)=e^{-(y-z)^{2} / x}+e^{-(y+z)^{2} / x}, \\
H(y, x)=y e^{-y^{2} / x} .
\end{gathered}
$$

The variable $x \in[0,1]$ sets the linear scale such that the whole interval $[0,1]$ corresponds to sites $i=1, \ldots, L$ in the TASEP. Second, for mapping onto densities via related local chemical potentials we also need [9]

$$
\begin{gathered}
I(y, x, \mu)=e^{-(2 \mu-y)^{2} / x}, \\
J(y, z, x, \mu)=e^{-(2 \mu+y-z)^{2} / x}, \\
K(y, x, \mu)=e^{-(2 \mu+y)^{2} / x},
\end{gathered}
$$

where $\mu$ is a rescaled local density [ $\mu_{p}$ in Eq. (15) below]; for the rescaling see (20) and Ref. [27].

For the FTASEP, instead of $F(y, x)$ in the TASEP we use at the input end of the lattice the slowly decaying Pareto form,

$$
P(y, x, a)=(1+y / \sqrt{x})^{-a-1} .
$$

This functional form is chosen to mimic a wide distribution of input rates, and the factor $1 / \sqrt{x}$ in the denominator is included for similar passage from $x=1$ to $x=1 / k$ with the functions $F(y, x)$ for the TASEP and $P(y, x, a)$ for the FTASEP. We also note that the derivative with respect to $y$ of $P(y, x, a)$ is of the same form but with larger $a$.

We then define the (unnormalized) effective partition function for a system consisting of $k$ consecutive intervals 
of (for simplicity) equal length as (cf. Refs. [9] and [27])

$$
\begin{aligned}
Z_{k}= & \int_{0}^{\infty} d y_{1} \int_{0}^{\infty} d y_{2} \ldots \int_{0}^{\infty} d y_{k-1} \int_{-\infty}^{\infty} d \mu_{1} \int_{-\infty}^{\infty} d \mu_{2} \ldots \\
& \times \int_{-\infty}^{\infty} d \mu_{k} W_{k}\left(y_{1}, y_{2}, \ldots, y_{k-1}, \mu_{1}, \mu_{2}, \ldots, \mu_{k}, x\right),
\end{aligned}
$$

where suppressing the parameter $x$ (or setting $x=1$, see below), we have for the TASEP,

$$
\begin{aligned}
W_{k}(\cdots)= & F\left(y_{1}\right)\left[\prod_{p=1}^{k-2} G\left(y_{p}, y_{p+1}\right)\right] H\left(y_{k-1}\right) \\
& \times I\left(y_{1}, \mu_{1}\right)\left[\prod_{p=2}^{k-1} J\left(y_{p-1}, y_{p}, \mu_{p}\right)\right] K\left(y_{k-1}, \mu_{k}\right) .
\end{aligned}
$$

For the ordinary TASEP this partition function is valid for the point $\alpha=\beta=1$, i.e., in the phase diagram deep inside the maximal current phase.

For the FTASEP with a given value of $a$, still suppressing dependence on $x$, we replace in Eq. (15) above $F\left(y_{1}\right) \rightarrow$ $P\left(y_{1}, a\right)$ and use the notations $W_{k} \rightarrow W_{k}^{a}$ and $Z_{k} \rightarrow Z_{k}^{a}$. For the FTASEP we use this for the case where Eq. (7) is valid. Physically, the choices made above lead to an effective model where the heavy-tail fluctuations at the input end of the lattice in the FTASEP get propagated through the system by the TASEP dynamics.

The integration in Eq. (15) over $\mu_{p}$ variables means that also noninteger values of the corresponding occupation numbers $n_{p}$ are included in averaging, which is not a problem as long as the system size is large and the location $p$ contains a large number of lattice sites [27].

One can calculate the integrals in Eq. (15) for a selected constant value of $x$ and from it get the partition function and, e.g., averages $\left\langle\mu_{p}^{v}\right\rangle$ for an other value of $x$ by a simple change in integration variables; in the actual calculations we first set $x=1$. Note also that it is possible to let in all of the above $x$ depending on $p$ with $\sum_{p} x_{p}=1$ such that $x_{p} L$ is the length of a fraction $x_{p}$ of the system size $L$ for each $p$.

As an example, one might set $x=1 / k=1 / L$, where $L$ is the number of sites in the exclusion process, and then use the connection $[9,27]$,

$$
\left\langle\mu_{p}\right\rangle \sqrt{L}=\left\langle n_{p}\right\rangle-L x / 2
$$

to obtain

$$
\left\langle n_{p}\right\rangle=1 / 2+\left\langle\mu_{p}\right\rangle \sqrt{L},
$$

with the interpretation that $\left\langle n_{p}\right\rangle$ is the average occupation number of a single site. In practice one can perform a calculation with $x=1$ from which

$$
\left\langle\mu_{p}\right\rangle=\left\langle\mu_{p}\right\rangle_{x=1} \sqrt{x}
$$

In any case, with $L=m k=m / x$ the local particle density $\rho_{p}$ in section $p=1,2, \ldots, k$ from Eq. (17) is written as

$$
\rho_{p}=\frac{\left\langle n_{p}\right\rangle}{m}=\frac{1}{2}+\sqrt{\frac{k}{m}}\left\langle\mu_{p}\right\rangle
$$

and

$$
\rho_{p}^{2}=\frac{\left\langle n_{p}^{2}\right\rangle}{m^{2}}=\frac{1}{4}+\sqrt{\frac{k}{m}}\left\langle\mu_{p}\right\rangle+\frac{k}{m}\left\langle\mu_{p}^{2}\right\rangle
$$

\section{A. The TASEP}

To compare with, we first consider the model for the standard TASEP. Then for $x=1$,

$$
Z_{k}(x=1)=\frac{\pi^{k-1 / 2}}{2^{k+2} k^{3 / 2}},
$$

and $Z_{k}(x)=x^{k-1 / 2} Z_{k}(x=1)$. Expectation values of specific interest (see later) are for the first site $(p=1)$ and last site $(p=k)$ as follows:

$$
\begin{gathered}
\left\langle\mu_{1}\right\rangle_{x=1}=-\left\langle\mu_{k}\right\rangle_{x=1}=[(1-1 / k) / \pi]^{1 / 2}, \\
\left\langle\mu_{1}^{2}\right\rangle_{x=1}=\left\langle\mu_{k}^{2}\right\rangle_{x=1}=1 / 2-3 / 8 k .
\end{gathered}
$$
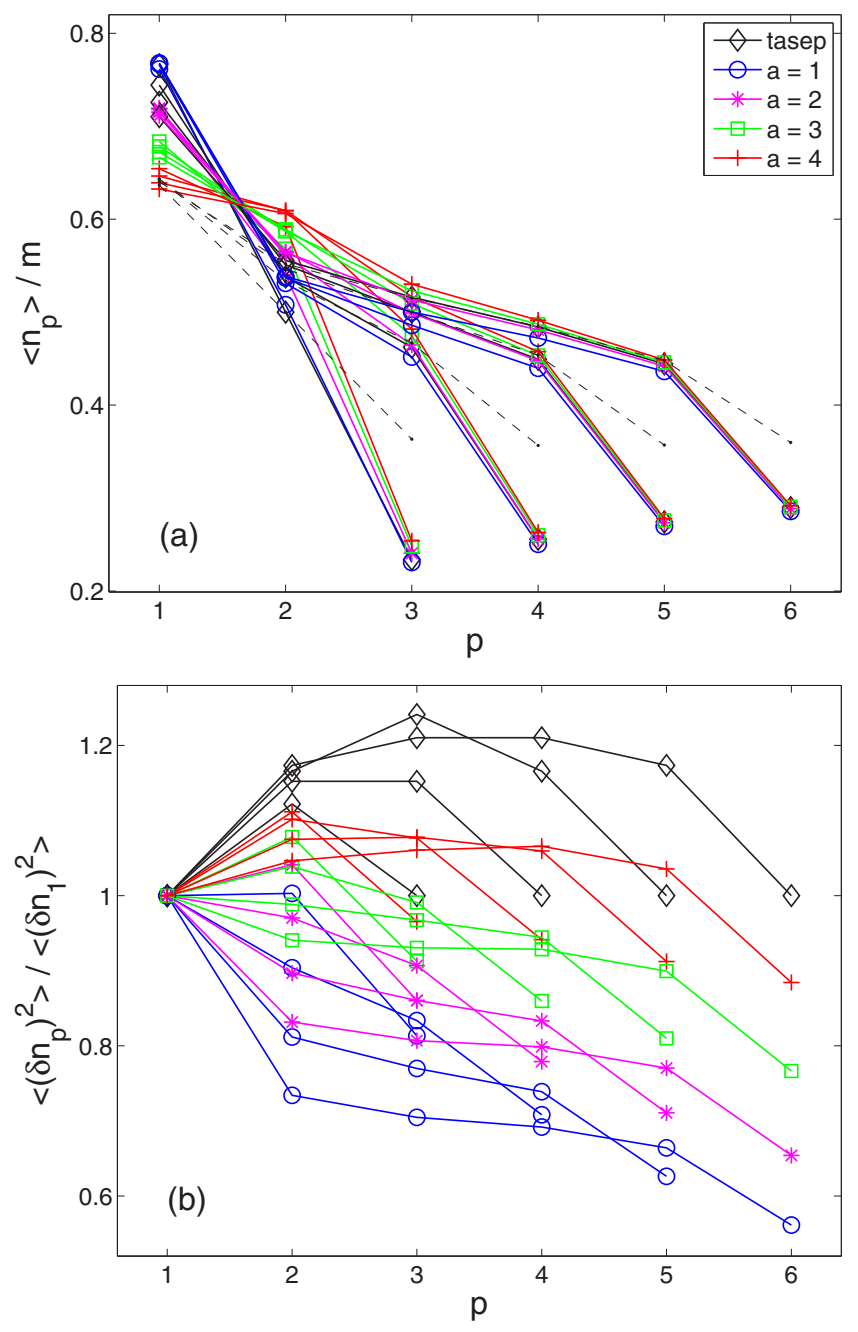

FIG. 5. (Color online) (a) Local particle density and (b) its variance for the TASEP and the FTASEP, from left to right $L=9,16,25,36$. The curves for $L=9,16,25,36$ end at $p=3-6$, respectively. The dashed lines in (a) show the exact predictions for the TASEP. 
For $x=1 / k=1 / L$ we would have $\left\langle\mu_{p}\right\rangle=\sqrt{x}\left\langle\mu_{p}\right\rangle_{x=1}$ and $\left\langle\mu_{p}^{2}\right\rangle=x\left\langle\mu_{p}^{2}\right\rangle_{x=1}$ for all $1 \leqslant p \leqslant k$. The formulas corresponding to those in Eqs. (23) and (24) for sites $1<p<k$ become more complicated and are not shown here. In terms of lattice site occupation we get for $x=1 / k=1 / L$,

$$
\begin{gathered}
\left\langle n_{1}\right\rangle=1 / 2+[(1-1 / k) / \pi]^{1 / 2}, \\
\left\langle\left(\delta n_{1}\right)^{2}\right\rangle=(1 / 2-1 / \pi)-(3 / 8-1 / \pi) / k,
\end{gathered}
$$

where $\left\langle\left(\delta n_{1}\right)^{2}\right\rangle \equiv\left\langle\left(n_{1}-\left\langle n_{1}\right\rangle\right)^{2}\right\rangle$. By symmetry in the TASEP with $\alpha=\beta=1$, we have $\left\langle n_{k}\right\rangle=1-\left\langle n_{1}\right\rangle$ and $\left\langle\left(\delta n_{k}\right)^{2}\right\rangle=$ $\left\langle\left(\delta n_{1}\right)^{2}\right\rangle$.

Related analytical and numerical results for a certain kind of division of the lattice into large segments for the TASEP with $\alpha=1=\beta$ can be found in Ref. [9]. The theory becomes exact for large $L$ and $m$ only [9,27], and then $\left\langle n_{p}\right\rangle \in[0,1]$. We note that in the interpretation $L=k$ we would have $m=1$ and for $k \rightarrow \infty$ then from the formulas above we find $\left\langle n_{1}\right\rangle \rightarrow 1 / 2+1 / \sqrt{\pi} \approx 1.064>1$ and $\left\langle n_{k}\right\rangle \rightarrow$ $1 / 2-1 / \sqrt{\pi} \approx-0.064<0$.

\section{B. The FTASEP}

Also for the FTASEP there are a few cases with certain values of $a, p, k$, and some $\left\langle\mu_{p}^{v}\right\rangle$ for which the partition function can be expressed analytically in terms of, e.g., hypergeometric functions and their special cases, such as the exponential integral and the Dawson integral, the actual formula given by, e.g., Mathematica depending on the order of integrals. Most of such expressions are cumbersome and difficult to use and do not provide much physical insight, see the Appendix. It is much more useful to express some of the results with the integral over $y_{1}$ left undone. Then, for the first site $p=1$ with $L=k$ for simplicity [see Eq. (19)], we find

$$
\left\langle\mu_{1}\right\rangle=\frac{\sqrt{1 / k}}{z_{k}^{a}} \int_{0}^{\infty} \frac{y^{2} e^{-y^{2} /(k-1)}}{2(1+y)^{a+1}} d y,
$$

and

$$
\left\langle\mu_{1}^{2}\right\rangle=\frac{1 / k}{z_{k}^{a}} \int_{0}^{\infty} \frac{y\left(1+2 y^{2}\right) e^{-y^{2} /(k-1)}}{8(1+y)^{a+1}} d y,
$$

where

$$
z_{k}^{a}=\int_{0}^{\infty} \frac{y e^{-y^{2} /(k-1)}}{(1+y)^{a+1}} d y .
$$

In Eqs. (27)-(29) some dependence on $k$ has been canceled, see the Appendix for detailed formulas. Note the dependence on the system size $L=k$ in the decay parameter of the integrands.

These integrals are easy to calculate accurately by numerical integration, whereas the original $\left(k^{2}-k\right)$-dimensional integrals with the integrand proportional to factors $\exp \left[-2 y_{p}^{2}\right]$ and $\exp \left[-2 y_{p} y_{p+1}\right]$ become prohibitively difficult to integrate numerically for $k>4$.

From the point of view of the propagation of fluctuations, the site at the end of the system $(p=k)$ is of interest. For the variance at $p=k$ we need, e.g.,

$$
\left\langle\mu_{k}^{2}\right\rangle=\frac{1 / \sqrt{k}}{z_{k}^{a}} \int_{0}^{\infty} \frac{y\left(c_{k}+2 y^{2}\right) e^{-y^{2} /(k-1)}}{8(1+y)^{a+1}} d y,
$$

where $c_{k}=(k-1)(4 k-7)$ replaces unity in Eq. (28) and

$$
\left\langle\mu_{k}\right\rangle=\frac{1 / k}{z_{k}^{a}} \frac{1}{2 \sqrt{\pi}(k-1)^{3 / 2}(k-2)^{1 / 2}} \int_{0}^{\infty} \frac{\left[b_{k} y+\sqrt{b_{k} \pi}\left(y^{2}+b_{k} / 2\right) e^{y^{2} / b_{k}} \operatorname{erf}\left(y / \sqrt{b_{k}}\right)\right] e^{-y^{2} /(k-1)}}{(1+y)^{a+1}} d y,
$$

where $b_{k}=(k-2)(k-1)$. With the error function being defined as an integral, Eq. (31) is a two-dimensional integral.

\section{RESULTS OBTAINED BY NUMERICAL INTEGRATION OF THE PARTITION FUNCTION}

We next turn to results obtained by computing numerically the partition function and averages for the local densities $\left\langle n_{p}\right\rangle$ and their fluctuations based on Eq. (15) for the TASEP and the FTASEP. For this, we start by computing $\left\langle\mu_{p}\right\rangle_{x=1}$ and $\left\langle\mu_{p}^{2}\right\rangle_{x=1}$ for the FTASEP with given values of $a$, where $p=1, \ldots, k$ with $k=3-6$. For the TASEP, the integrations involved can be performed exactly as shown in Sec. IV, whereas for the FTASEP some numerical integration is needed in most cases. To study large systems of $L$ sites, we then set $m=L / k$ with $x=1 / k$ and $\left\langle\mu_{p}\right\rangle=\left\langle\mu_{p}\right\rangle_{x=1} \sqrt{x}$ from Eq. (19), so local densities are

$$
\frac{\left\langle n_{p}\right\rangle}{m}=\frac{1}{2}+\sqrt{\frac{k}{m}}\left\langle\mu_{p}\right\rangle,
$$

with $m$ being the number of lattice sites in interval $p$.
In Figs. 5 and 6 we show the local particle density and its variance for the TASEP and the FTASEP for a convenient selection of system sizes. The dashed lines display the local particle density calculated from the exact formula for singlesite occupation numbers in the TASEP with $\alpha=\beta=1$ given in Eq. (48) of Ref. [3]. Comparing the TASEP result for $\left\langle n_{p}\right\rangle / m$ by numerical integration in Figs. 5(a) and 6(a) with the exact result for the TASEP, we conclude that numerical integration works well for large system sizes, and we can proceed to study fluctuations.

To make the structure of the curves in Figs. 5(b) and 6(b) more visible for all values of $a$ we have normalized the data by dividing $\left\langle\left(\delta n_{p}\right)^{2}\right\rangle$ by the variance $\left\langle\left(\delta n_{1}\right)^{2}\right\rangle$. The data for the normalized variance show that the fluctuation profile in the FTASEP differs from that in the TASEP more than could be expected from the local density data. It is even a monotonically decreasing function of the distance from the input site for $a=$ $1-3$. For increasing $a$ it approaches the symmetric form of the fluctuation profile of the TASEP. The absolute unnormalized values (not shown) of the variance increase fast for decreasing $a$ as was seen in the simulation data of Fig. 1(a) in the preceding 

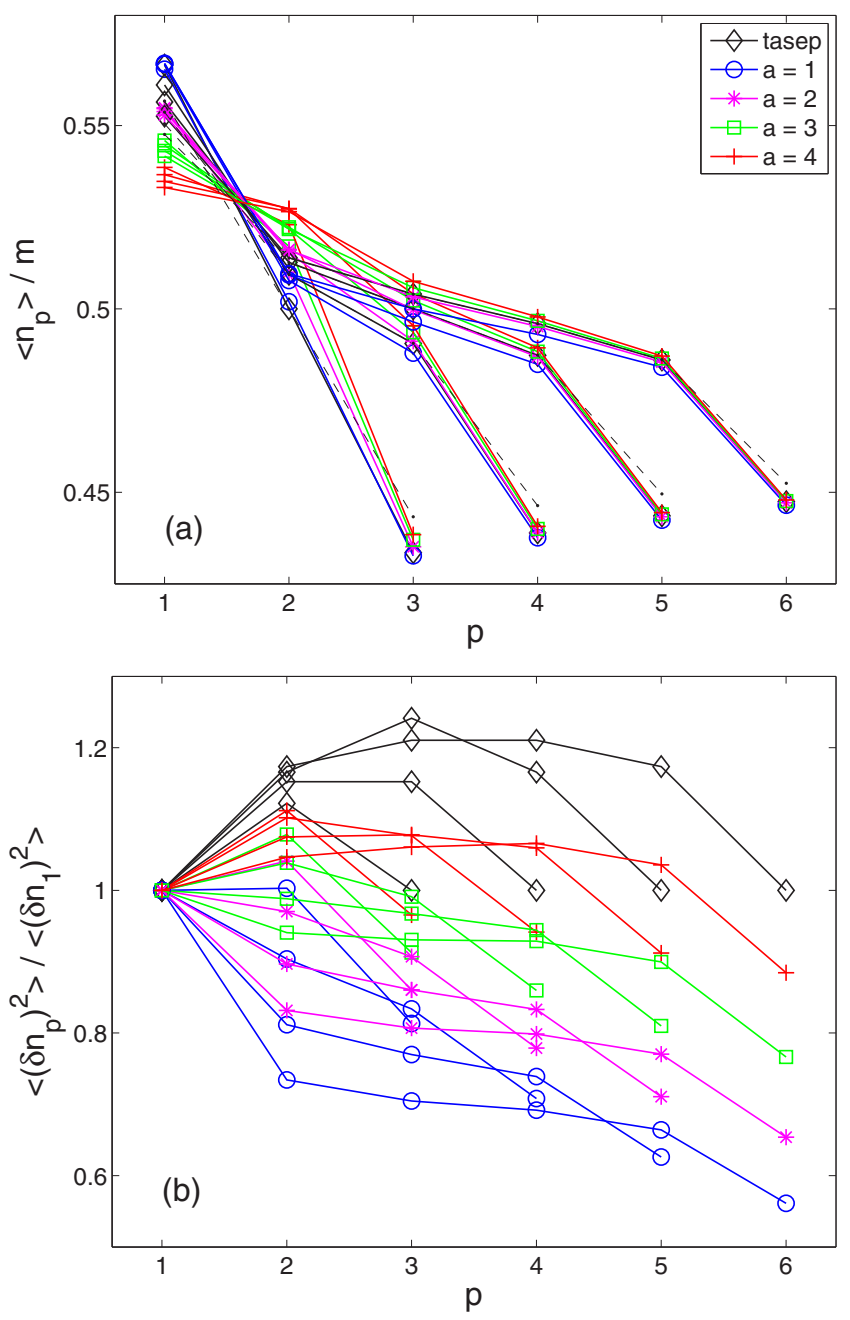

FIG. 6. (Color online) The same as Fig. 5 but with $L=$ $144,256,400,576$. The curves for $L=144,256,400,576$ end at $p=3-6$, respectively.

section. Note that the data in Figs. 5(b) and 6(b) are due to the normalization the same, but the absolute values of $\left\langle\left(\delta n_{p}\right)^{2}\right\rangle$ differ by a factor of 16 , being larger in the first case.

It is of interest to compare the theoretical $(T)$ results obtained by numerical integrations with those obtained by simulations $(S)$ for the FTASEP. We continue to use the simulation data with $L=500$. Now in the effective theoretical model there are different ways to treat the first site of the lattice. In Fig. 7 we show the result of three choices: the length of the first interval in integrations $m_{1}=1,10,84$ lattice units and the rest of the lattice is divided evenly such that the total number of intervals is 6 , i.e., $p=1,2, \ldots, 6$. The case of $m_{1}=84$ corresponds to the equal division of the lattice. The conversion from chemical potentials to densities becomes $\left\langle n_{p}\right\rangle / m_{p}=1 / 2+\left\langle\mu_{p}\right\rangle_{x_{p}=1} / \sqrt{m_{p}}$. The small values of $m_{1}$ from the theoretical point of view $(T)$ have the problem that the intervals should be "macroscopic" parts of the lattice, whereas in the simulations $(S)$ only the first site is fed by a non-Poissonian source. Therefore, for each $a=2-4$ we show the comparison for $m_{1}=1,10,84$. For the TASEP (not shown)

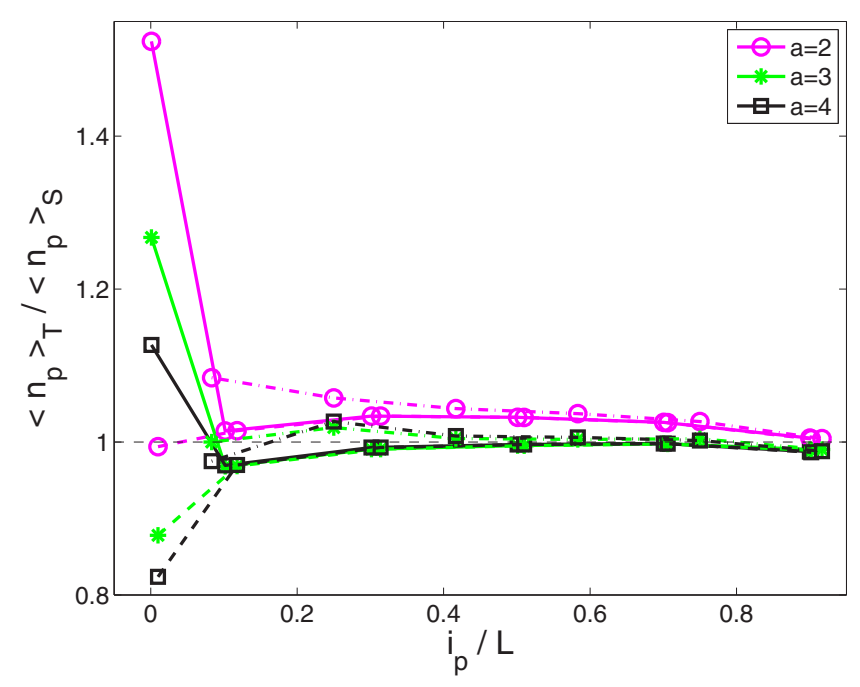

FIG. 7. (Color online) Local particle density in theoretical computation $(T)$ and simulation $(S)$ compared for $a=2-4$ (shown by circles, stars, and squares, respectively) for three widths of the first interval $m_{1}=1,10,84$ (shown by full lines, dashed lines, and dashed-dotted lines, respectively). The horizontal axis is defined here in such a way that the data points correspond to midpoints of the intervals at $i=i_{p}$.

with an evenly divided system the agreement is perfect, i.e., $\left\langle n_{p}\right\rangle_{T} /\left\langle n_{p}\right\rangle_{S}=1$. In simulations for $a=1$ the density is so low that no reasonable comparison can be made. Note that we have not attempted to fine-tune the density of the first interval in the simulation to correspond to that of the theoretical calculation. We conclude that the theoretical and the simulation approaches agree reasonably well. However, the agreement does depend on $a$ for the given choice of $m_{1}$ since for different values of $a$ the correlations in the input noise die out in different length scales.

\section{CONCLUSIONS}

To summarize, we used continuous-time Monte Carlo simulation and numerical evaluation of the partition function to study the effect of non-Poissonian power-law-governed feeding on the totally asymmetric exclusion process. This model we call the FTASEP. In particular we studied local density fluctuations across the system to characterize propagation of the fluctuations through the system. Depending on the parameter $a$ defining the decay of the waiting-time distribution, we observe simple density-controlled low-density behavior for small values of $a$, complex behavior for intermediate values of $a$, and the approach of the FTASEP to the TASEP for large values of $a$. It would be of interest to extend the study of the combined effect of non-Poissonian feeding and finite size also to the statistics of current fluctuations.

\section{APPENDIX: SOME ANALYTICAL RESULTS}

For the FTASEP for some values of $a$ the partition function $Z_{k}^{a}$ can be expressed in a closed form in terms of special 
functions. For example,

$$
Z_{5}^{1}(x=1)=\frac{\pi^{4}}{4096}\left\{4+2 \sqrt{\pi}\left[5 F\left(2^{-1}\right)-3\right]-e^{-1 / 4} \operatorname{Ei}\left(4^{-1}\right)\right\},
$$

where

$$
F(x)=\int_{0}^{x} e^{t^{2}-x^{2}} d t \quad \text { and } \quad \operatorname{Ei}(x)=-\int_{-x}^{\infty} t^{-1} e^{-t} d t .
$$

It turns out that

$$
Z_{k}^{a}(x=1)=\frac{\pi^{k-1}}{2^{k}(k-1)^{3 / 2}} \int_{0}^{\infty} \frac{y e^{-y^{2} /(k-1)}}{(1+y)^{a+1}} d y,
$$

and the unnormalized expectation values are

$$
\left\langle\mu_{1}\right\rangle_{x=1} Z_{k}^{a}(x=1)=\frac{\pi^{k-1}}{8(k-1)^{3 / 2}} \int_{0}^{\infty} \frac{y^{2} e^{-y^{2} /(k-1)}}{(1+y)^{a+1}} d y,
$$

and

$$
\begin{aligned}
\left\langle\mu_{1}^{2}\right\rangle_{x=1} Z_{k}^{a}(x=1)= & \frac{\pi^{k-1}}{32(k-1)^{3 / 2}} \\
& \times \int_{0}^{\infty} \frac{y\left(1+2 y^{2}\right) e^{-y^{2} /(k-1)}}{(1+y)^{a+1}} d y .
\end{aligned}
$$

Corresponding formulas can be obtained for other sites of $p$. The most interesting and useful, i.e., relatively simple, result is obtained for $p=k$, see Sec. IV B.
[1] F. Spitzer, Interaction of Markov processes, Adv. Math. 5, 246 (1970).

[2] H. Spohn, Large Scale Dynamics of Interacting Particles (Springer, New York, 1991).

[3] B. Derrida, E. Domany, and J. L. Lebowitz, An exact solution of a one-dimensional asymmetric exclusion model with open boundaries, J. Stat. Phys. 69, 667 (1992).

[4] B. Schmittmann and R. K. P. Zia, in Phase Transitions and Critical Phenomena, edited by C. Domb and J. Lebowitz (Academic, London, 2001), Vol. 17.

[5] M. R. Evans and T. Hanney, Nonequilibrium statistical mechanics of the zero-range process and related processes, J. Phys. A 38, R195 (2005).

[6] T. Chou, K. Mallick, and R. K. P. Zia, Non-equilibrium statistical mechanics: From a paradigmatic model to biological transport, Rep. Prog. Phys. 74, 116601 (2011).

[7] T. Antal and G. M. Schütz, Asymmetric exclusion process with next-nearest-neighbor interaction: Some comments on traffic flow and a nonequilibrium reentrance transition, Phys. Rev. E 62, 83 (2000).

[8] G. M. Schütz, in Phase Transitions and Critical Phenomena, edited by C. Domb and J. Lebowitz (Academic, London, 2001), Vol. 19.

[9] B. Derrida, C. Enaud, and J. L. Lebowitz, The asymmetric exclusion process and brownian excursions, J. Stat. Phys. 115, 365 (2004).

[10] M. R. Evans, T. Hanney, and S. N. Majumdar, Interaction-driven real-space condensation, Phys. Rev. Lett. 97, 010602 (2006).

[11] O. Hirschberg, D. Mukamel, and G. M. Schütz, Condensation in temporally correlated zero-range dynamics, Phys. Rev. Lett. 103, 090602 (2009).

[12] A. Gabel, P. L. Krapivsky, and S. Redner, Facilitated asymmetric exclusion, Phys. Rev. Lett. 105, 210603 (2010).

[13] A. Gabel and S. Redner, Dynamic singularities in cooperative exclusion, J. Stat. Mech. (2011) P06008.

[14] R. K. P. Zia, J. J. Dong, and B. Schmittmann, Modeling translation in protein synthesis with tasep: A tutorial and recent developments, J. Stat. Phys. 144, 405 (2011).

[15] B. Waclaw and M. R. Evans, Explosive condensation in a mass transport model, Phys. Rev. Lett. 108, 070601 (2012).
[16] S. Grosskinsky, F. Redig, and K. Vafayi, Dynamics of condensation in the symmetric inclusion process, Electron. J. Probab. 18, 1 (2013).

[17] O. Hirschberg, D. Mukamel, and G. M. Schütz, Emergent motion of condensates in mass-transport models, Phys. Rev. E 87, 052116 (2013).

[18] P. L. Krapivsky, Dynamics of repulsion processes, J. Stat. Mech. (2013) P06012.

[19] J. Dong, S. Klumpp, and R. K. P. Zia, Entrainment and unit velocity: Surprises in accelerated exclusion process, Phys. Rev. Lett. 109, 130602 (2012).

[20] J. Dong, S. Klumpp, and R. K. P. Zia, Mass transport perspective on an accelerated exclusion process: Analysis of augmented current and unit-velocity phases, Phys. Rev. E 87, 022146 (2013).

[21] J. Merikoski, Accelerated transport and growth with symmetrized dynamics, Phys. Rev. E 88, 062137 (2013).

[22] D. A. Adams, B. Schmittmann, and R. K. P. Zia, Far-fromequilibrium transort with constrained resources, J. Stat. Mech. (2008) P06009.

[23] V. Popkov, M. Salerno, and G. M. Schütz, Asymmetric simple exclusion process with periodic boundary driving, Phys. Rev. E 78, 011122 (2008).

[24] R. J. Concannon and R. A. Blythe, Spatiotemporally complete condensation in a non-poissonian exclusion process, Phys. Rev. Lett. 112, 050603 (2014).

[25] A. B. Bortz, M. H. Kalos, and J. L. Lebowitz, A new algorithm for monte carlo simulation of ising spin systems, J. Comput. Phys. 17, 10 (1975).

[26] D. T. Gillespie, A general method for numerically simulating the stochastic time evolution of coupled chemical reactions, J. Comput. Phys. 22, 403 (1976).

[27] Note that the meanings of the chemical potential in discussions of mass transport models are often different from its standard meaning in equilibrium statistical mechanics, see, e.g., Refs. [5,9]. In the present context $\mu_{p}$ 's become essentially rescaled local densities. More technically, in Ref. [9] it is shown that the local particle density $\rho(x)$ in the maximum current phase can be written as $\rho(x) \simeq[\dot{B}(x)+\dot{Y}(x)] / 2 \sqrt{L}+1 / 2$, where $\dot{B}$ is the derivative of a Brownian path and $\dot{Y}$ is the derivative 
of a Brownian excursion, these being independent processes. Then, integrating over a section $\left[x_{p-1}, x_{p}\right]$ of the system, one finds $L \int[\rho(x)-1 / 2] d x \simeq \sum_{i}\left(n_{i}-1 / 2\right)=\mu_{p} \sqrt{L}$ where the summation over lattice sites $i$ runs from $L x_{p-1}$ to $L x_{p}$, valid for large $L$ and large $L x_{p}$. This connection is obtained in Ref. [9] by using the (nowadays standard) matrix ansatz method for the TASEP.

[28] L. C. G. Rogers and D. Williams, in Diffusions, Markov Processes and Martingales (Cambridge University Press, Cambridge, UK, 2000), Vol. 2, p. 421. 